\title{
Ermittlung von Anforderungen an eine Anwendungsfall-Spezifische Einführung Agiler Ansätze - Erkenntnisse aus der Anwendung des Agile-Stage-Gate Hybrids
}

Jonas Heimicke, Ahmed Spahic, Luis Bramato, Albert Albers

Produzierende Unternehmen, die traditionelle Produktentwicklungsmethoden mit linearen und rigiden Prozessen nutzen, sind zunehmend weniger in der Lage, den Veränderungen in Märkten und Technologien gerecht zu werden. Der klassische Stage-Gate Prozesses nach Cooper lässt kein adäquates Reagieren auf diese Veränderungen zu, sodass es zu Verzögerungen in der Entwicklung und späten Änderungen am Produkt im weiteren Projektverlauf kommt. Aus diesem Grund werden agile Methoden innerhalb etablierter plangetriebener Ansätze integriert, um durch kurzfristige und wiederkehrende Planungs-aktivitäten und ein iteratives Vorgehen in der Entwicklung eine schnellere Anpassung an mögliche Veränderungen während der Projektdurchführung zu ermöglichen. Die Anwendung agiler Methoden, die aus der Softwareentwicklung entstammen, kann jedoch nicht ohne Anpassungen erfolgen, da sich der Entwicklungsprozess physischer Produkte beispielsweise hinsichtlich der Modularisierung des Gesamtsystems in kleinere Inkremente deutlich von der Vorgehensweise in der Softwareentwicklung unterscheidet. Um die Einführung agiler Methoden unternehmensspezifisch und zweckmäßig gestalten zu können, soll die Basis für einen unternehmensspezifischen Problemlösungsprozess gelegt werden, mit dem Agilität in die Prozesse eingeführt werden kann. Dazu soll herausgefunden werden, inwieweit Entwickler aus der Praxis den Bedarf für eine individuelle Einführung von Agilität sehen. Dazu werden Interviews mit Experten aus Unternehmen geführt, um die Implementierung agiler Methoden im Rahmen der physischen Produktentwicklung in der Praxis zu hinterfragen und zukünftige Forschungspotentiale zu explizieren. Daraus werden Hypothesen hinsichtlich einer individuellen Einführung von Agilität in die Entwicklungsprozesse produzierender Unternehmen abgeleitet und in einer quantitativen Umfrage überprüft. Die Ergebnisse zeigen eindeutig den Bedarf für eine individuelle Einführung von Agilität in den jeweiligen Anwendungsfall.

Keywords: Agilität, Agile Produktentwicklung, Produktentwicklungsprozess, Interviewstudie. 


\section{Einleitung}

Unternehmen und Organisationseinheiten, die traditionelle Produktentwicklungsmethoden mit linearen und rigiden Prozessen nutzen, sind heute immer weniger in der Lage, sich den Veränderungen in Märkten und Technologien rechtzeitigt anzupassen. (Cooper \& Sommer, 2018) Aus diesem Grund werden zunehmend agile Methoden innerhalb plangetriebener Ansätze integriert (Atzberger, Nicklas, Schrof, Weiss \& Paetzold, 2020), um sich durch kurzfristigere und wiederkehrende Planung und einem iterativen Vorgehen schneller an mögliche Veränderungen während der Projektdurchführung anzupassen (Heimicke, Kaiser \& Albers, 2021; Wessels et al., 2019). Dabei wird der Entwicklungsprozess mittels entsprechend adäquatem Planungsaufwand in kleine Abschnitte unterteilt, innerhalb derer binnen eines kurzen Zeitraums ein validierbares Ergebnis bzw. ein funktionsfähiges Produktinkrement erzeugt wird, dieses validiert wird und daraus wiederum konkrete Ziele und Anforderungen für den weiteren Projektverlauf gewonnen werden können (Wessels et al., 2019). Dabei werden zumeist agile Techniken eingesetzt, die ihren Ursprung in der Softwareentwicklung haben (Atzberger et al., 2020; Heimicke, Kaiser \& Albers, 2021). Die Anwendung agiler Methoden aus der Softwareentwicklung sollte jedoch in der Entwicklung mechatronischer Systeme nicht ohne gezielte Anpassungen erfolgen (Goevert, Heimicke, Lindemann \& Albers, 2019), da sich der Entwicklungsprozess physischer Produkte beispielsweise hinsichtlich der Aufteilbarkeit in kleinere Inkremente (Ovesen, 2012) deutlich von der Softwareentwicklung unterscheidet (Cooper, 2014). Die gezielte Aufbereitung agiler Elemente für den jeweiligen Entwicklungskontext und deren spezifische Einführung ist die Grundlage für eine nachhaltige Prozessverbesserung (Diebold, Küpper \& Zehler, 2015; Heimicke, Dühr, Krüger, Ng \& Albers, 2021). Im vorliegenden Beitrag werden zu diesem Zweck grundlegende Anforderungen an die Einführung agiler Elemente in die Prozesse der Mechatroniksystementwicklung ermittelt.

\section{Stand der Forschung}

\section{Plangetriebene Vorgehensmodelle der Produktentwicklung}

Die VDI-Richtlinie 2221 definiert die Produktentwicklung als „interdisziplinärer Unternehmensprozess zur Entwicklung eines marktfähigen Produkts, basierend auf der Definition initialer Ziele und Anforderungen an das Produkt, die im Lauf des Prozesses kontinuierlich weiterentwickelt und iterativ angepasst werden“ (Richtlinie VDI 2221 Blatt 1). Generell lassen sich bestehende Vorgehensmodelle in plangetrieben, agil und 
hybrid unterteilen (Heimicke, Chen \& Albers, 2020; Naefe \& Luderich, 2020). Plangetriebene Vorgehensmodelle werden durch ihren sequentiellen Ablauf charakterisiert, weswegen sie häufig auch als Wasserfall-Modelle bezeichnet werden. Dabei werden die Anforderungen an das zu entwickelnde Produkt zu Beginn des Entwicklungsprojektes durch die Erstellung eines Lasten- und Pflichtenhefts definiert. Das gesamte Entwicklungsprojekt wird anschließend in einzelne Phasen aufgeteilt, die sequentiell bearbeitet werden und jeweils eine Überprüfung hinsichtlich Planabweichungen beinhalten. (Petersen \& Wohlin, 2010) Ein Beispiel ist der Stage-Gate Prozess nach Cooper, der die Produktentwicklungsschritte von der Ideengenerierung bis hin zur Implementierung umfasst und den Produktentwicklungsprozess in eine definierte Anzahl von Phasen und Meilensteinen unterteilt (Cooper, 1990).

\section{Agile und hybride Ansätze in der Produktentwicklung}

Agile und hybride Ansätze der Produktentwicklung basieren im Gegensatz zu plangetriebenen Vorgehensmodellen auf der Prämisse, dass sich ein Projektablauf vor Beginn der Entwicklungstätigkeiten nicht vollständig beschreiben und definieren lässt. Aus diesem Grund werden einzelne Projektschritte iterativ und in kurzen Zyklen geplant. (Naefe \& Luderich, 2020)

Ein agiles Vorgehensmodell, was zunächst in der Software-Entwicklung etabliert wurde und zunehmend auch Anwendung im Bereich der physischen Produktentwicklung findet, ist Scrum. Scrum basiert auf einer iterativ-inkrementellen Vorgehensweise, und besteht aus insgesamt drei Kategorien: Events/Meetings, Rollen sowie Artefakten (Schwaber \& Sutherland, 2017).

Hybride Vorgehensmodelle vereinen die Merkmale plangetriebener und agiler Ansätze (Heimicke et al., 2020). Ein Vertreter - der Agile-Stage-Gate Hybrid nach Cooper - verbindet auf operativer Ebene agile Elemente nach Scrum mit traditionellen, plangetriebenen Elementen auf taktischer und strategischer Ebene (vgl. Abbildung 1). (Cooper \& Sommer, 2018)

Die klassischen Projektphasen und Meilensteine dienen hierbei als Orientierung für die Kommunikation innerhalb eines Produktentwicklungsprojektes zwischen dem Projektteam auf der operativen Ebene und der strategischen Management-Ebene (Karlström \& Runeson, 2006). 


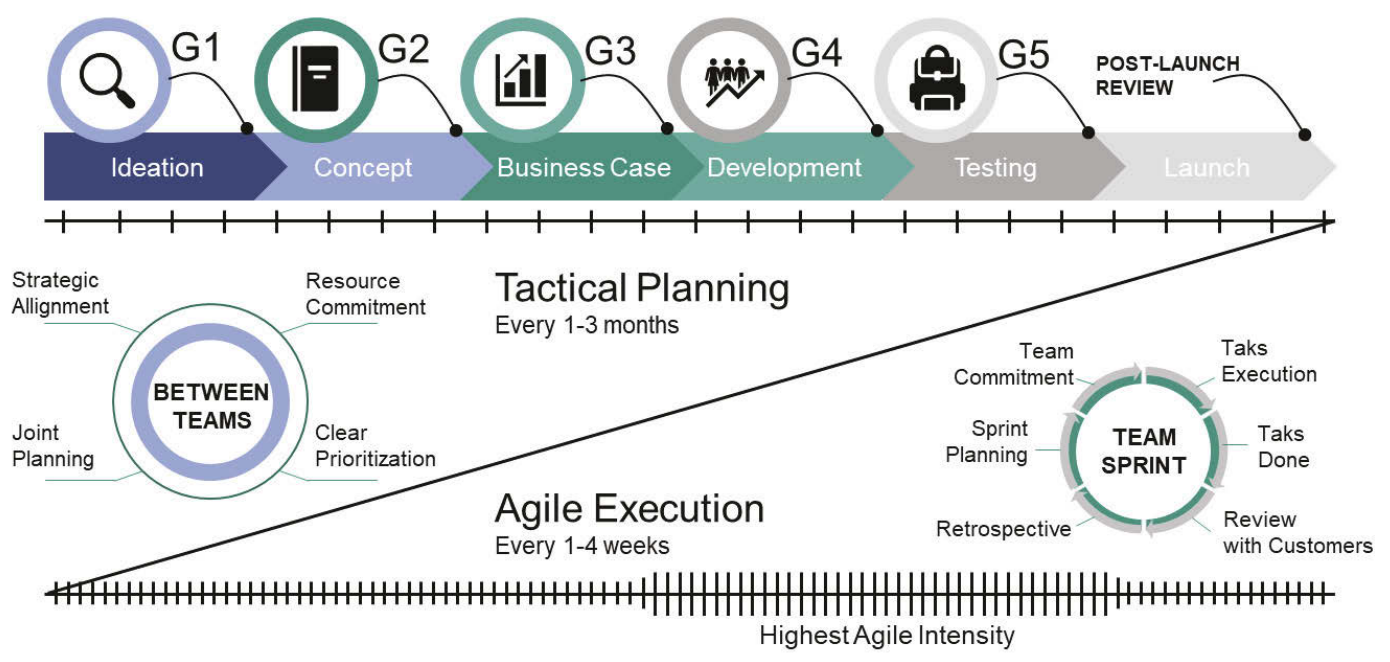

Abbildung 1: Übersicht Agile-Stage-Gate Hybrid (Cooper \& Sommer, 2018)

\section{Einführung agiler Ansätze in die Mechatroniksystementwicklung}

Die Hauptgründe für die Einführung von Agilität beziehen sich für Unternehmen meist auf Zeit, Volatilität und Prozessqualität (Theobald \& Diebold, 2018). Doch der Weg hin zu agilen Strukturen birgt viele Herausforderungen und erfordert kulturelle Veränderungen, welche für ein Team oder eine Organisation sehr schwierig sein können (Moreira, 2013).

Der ASD - Agile Systems Design-Ansatz „ist ein ganzheitlicher, strukturierender Ansatz zur agilen Entwicklung mechatronischer Systeme, sowie der zugehörigen Produktstrategie, Validierungssysteme und Produktionssysteme bestehend aus Denkweisen, Methoden und Prozessen der PGE - Produktgenerationsentwicklung" (Albers et al., 2018). Der Ansatz besteht aus insgesamt neun Grundprinzipien und schafft ein praktikables Gleichgewicht an strukturierenden und agilen Elementen, die in der Mechatroniksystementwicklung gezielt assistieren sollen (Albers et al., 2019). Zur Anwendungsfall-spezifischen Einführung agiler Elemente in die Prozesse der Mechatroniksystementwicklung wurde ein Framework entwickelt, dass die Elemente des ASD mittels eines Problemlösungsprozesses situations- und bedarfsgerecht einführt (Heimicke, Dühr, et al., 2021; Heimicke, Ng, Krüger \& Albers, 2021). 


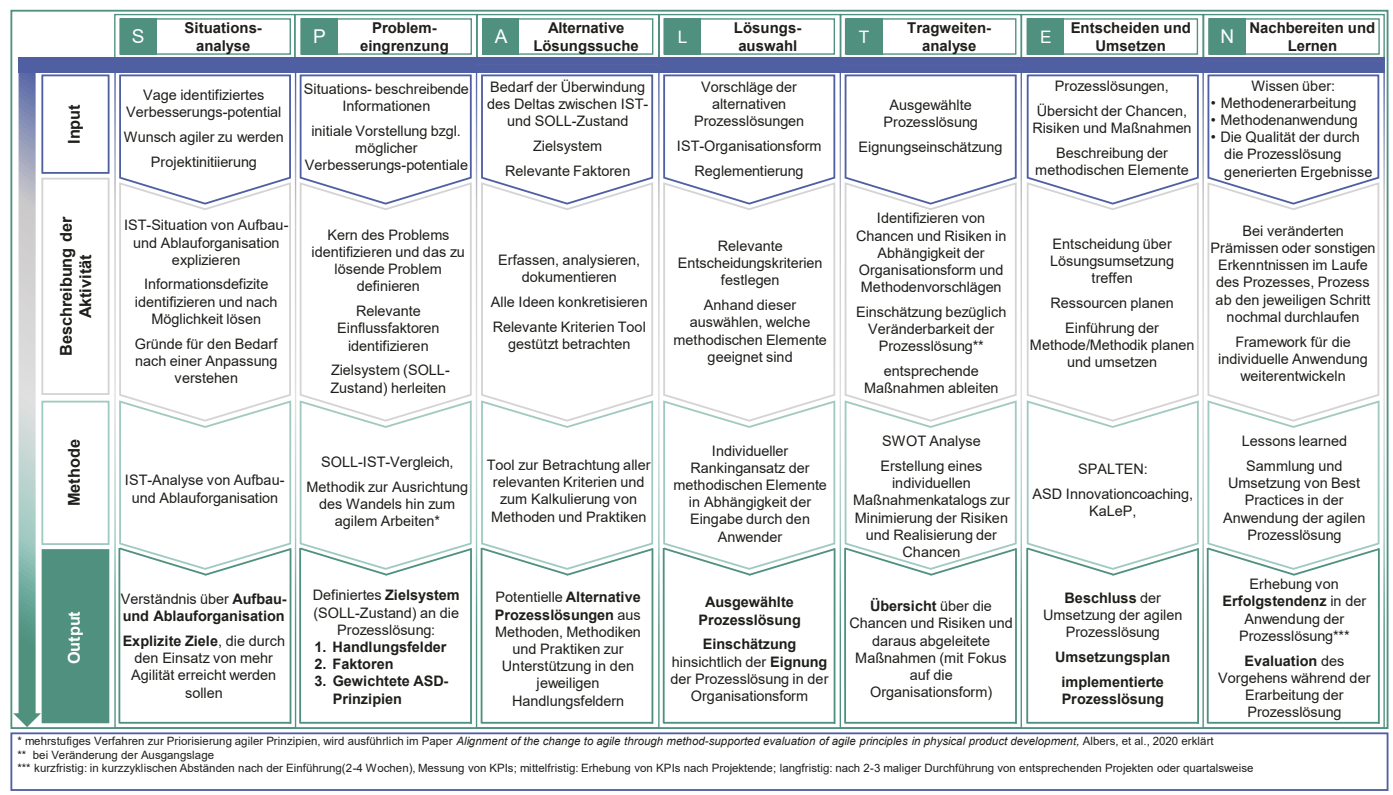

Abbildung 2: Framework zur situations- und bedarfsgerechten Einführung von Agilität

(Heimicke, Dühr, Krüger, Ng \& Albers, 2021)

Der Durchlauf des Problemlösungsprozesses ist durch verschiedene Aktivitäten gekennzeichnet, mittels derer schlussendlich aus einer Sammlung von über 130 agilen und plangetriebenen Elementen geeignete ausgewählt werden. Diese werden zu einer individuellen Prozesslösung kombiniert. Damit die ausgewählten Elemente das im jeweiligen Anwendungsfall tatsächlich vorliegende Verbesserungspotential adressieren, wird im ersten Teil des Frameworks die Situation erfasst und daraus aus 225 Faktoren 20 geeignete zur Optimierung ausgewählt. Die daraufhin vorgeschlagenen Elemente verbessern die jeweils ausgewählten Faktoren. (Heimicke, Ng, et al., 2021)

\section{Forschungsdesign}

Grundsätzlich besteht die Vermutung, dass die Einführung von Agilität als Problemlösungsprozess betrachtet werden kann, da die Ausgangslage zumeist unklar ist, die Vorstellung bezüglich des idealen SOLL-Zustands vage ist und der Weg vom IST- zum SOLL ebenfalls unklar und individuell ist, womit die grundlegenden Elemente eines Problems vorhanden sind (Richtlinie VDI 2221 Blatt 1). Die Einführung von agilen Ansätzen in die physische Produktentwicklung erfolgt jedoch in der Regel durch die anpassungsfreie Anwendung bestehender Ansätze. Ziel der Forschungsarbeit ist es, die Hypothese, 
dass die Einführung von Agilität ein Problemlösungsprozess ist, zu verifizieren und damit die Notwendigkeit einer methodischen Einführung von Agilität in die Entwicklungsprozesse produzierender Unternehmen zu motivieren. Dabei sollen folgende Hypothesen eingeschätzt werden:

- H1: Die Einführung von Agilität ist ein Problemlöseprozess.

- H2: Um Agilität erfolgreich in Entwicklungsprozesse zu implementieren, müssen die individuellen Ziele, die damit erreicht werden sollen, zunächst identifiziert werden.

- H3: Für eine erfolgreiche Einführung von Agilität muss für jeden Anwendungsfall ein individueller Ansatz entwickelt werden, der auf den im Entwicklungsumfeld vorhandenen Strukturen aufsetzt und gezielt agile Elemente in die Entwicklungsprozesse einführt.

- H4: Der Prozess Optimierungspotentiale zu identifizieren und Agilität in einem Unternehmen erfolgreich einzuführen, muss methodisch gestützt sein.

Um die genannten Hypothesen zu testen, lässt sich das Vorgehen in zwei Bereiche aufteilen: Aufbauend auf den Erkenntnissen aus der Literatur sollten identifizierte Herausforderungen und Potentiale durch die Durchführung einer qualitativen Studie in Form von Experteninterviews vertieft werden, um die Einführung agiler Methoden im Rahmen der physischen Produktentwicklung in der Praxis zu analysieren und neue Perspektiven zu generieren. Die Erkenntnisse dienten als Grundlage für die Durchführung einer quantitativen Studie, die das mengenmäßige Erfordernis für eine individuelle, methodische Einführung agiler Elemente in die Prozesse der Mechatroniksystementwicklung ermittelte. Dabei wurden mithilfe der Online-Umfrage-Applikation LimeSurvey für die Umfrage relevante Gruppen, also insbesondere agilitätserfahrene Produktentwickler, angefragt. Die Umfrage umfasste 6 Fragen. Die Verbreitung der Umfrage erfolgte sowohl über Karrierenetzwerke als auch über direkte, persönliche Anfragen. Das Erhebungsverfahren wurde anhand einer Likert-Skala mit vier Merkmalsausprägungen und der Möglichkeit keine Antwort zu geben durchgeführt. Teilnehmer, die bei einer Aussage „Keine Antwort" auswählten, wurden bei der jeweiligen prozentualen Auswertung nicht berücksichtigt. Haben bei einer Hypothese mehr als $70 \%$ der Teilnehmer mindestens die Ausprägung "Stimme eher zu“ gewählt, so verzeichnen die Autoren dies insgesamt als große Zustimmung der jeweiligen Aussage und erkennen diese im Kontext der vorliegenden Forschungsarbeit als verifiziert an. 


\section{Ergebnisse}

\section{Ergebnisse der qualitativen Untersuchung}

Der Leitfaden für die qualitative Untersuchung besteht aus sechs Themenbereichen mit insgesamt 21 Fragen. Im Themenbereich „Einführung agiler Elemente“ sind beispielsweise folgende Fragen aufgeführt:

1) Wurden agile Vorgehensmodelle bzw. Elemente allgemein etabliert oder wurden agile Vorgehensmodelle bzw. Elemente individuell auf den Unternehmenskontext angepasst?

2) Gab es während der Einführung agiler Vorgehensmodelle bzw. Elemente eine interne oder externe methodische Unterstützung?

Die qualitative Interviewstudie wurde auf Basis des erstellten Leitfadens durchgeführt. Dabei wurden die Interviews semistrukturiert durchgeführt, aufgezeichnet und anschließend transkribiert. Die Auswertung erfolgte nach der qualitativen Inhaltsanalyse nach MAYRING \& FRENZL (2014). Dabei wurden in Anlehnung an den Interview-Leitfaden Haupt- und Unterkategorien gebildet, in welche die einzelnen Textpassagen der transkribierten Interviews eingefügt wurden. Auf Basis der zwölf durchgeführten Interviews mit dreizehn Interviewpartnern, sind insgesamt drei Themenbereiche bezüglich der Einführung agiler Methoden in der physischen Produktentwicklung hervorzuheben. Die Interviewpartner sind dabei der Automobil-, Prozess-, Klimatechnik- sowie der Unternehmensberatungsbranche zuzuordnen. Erstens ist die Notwendigkeit einer individuellen Einführung agiler Methoden zu nennen. Dies bezieht auf die unternehmensspezifische und zielorientierte Anpassung agiler Methoden an die Anforderungen und Bedürfnisse des Unternehmens. Zweitens ist eine Top-Down-Awareness bei der Einführung agiler Methoden, ausgehend von der Geschäftsführung bzw. dem Top Management, als zentraler Erfolgsfaktor zu verfolgen. Eine Einführung, die von der operativen Ebene ohne Awareness der Top-Management-Ebene getrieben wird, wird als nicht effektiv und effizient empfunden, da die unterschiedliche Arbeitsweise und das unterschiedliche Mindset insbesondere an den Schnittstellen zwischen operativer, taktischer und strategischer Ebene zu großen Herausforderungen führen. Hierzu ist die Aussage des Interviewpartners 5 zu nennen, der als Projektleiter in der Automobilindustrie tätig ist:

„Aber es ist ganz klar: Es ist kontextabhängig. Es gibt keinen Blueprint. Den Blueprint, den es vielleicht gibt, kann man ja ganz einfach zusammenfassen: Das muss von oben nach unten gehen. Das heißt, das ist der einzige Blueprint für mich, den es gibt, das Management muss voll dahinterstehen und muss es verstehen. Das heißt, die sagen: "Wir verändern uns, 
wir führen das ein, wir müssen das Ganze auch unterstützen." Die mögen vielleicht auch noch am besten jemanden zur Verfügung stellen wie so eine Art Transformations-Manager, der sich dann kümmert extern oder intern mit entsprechenden Agile Coaches [...]"

Drittens ist eine methodengestützte Einführung agiler Methoden zu befürworten. In der Praxis werden hierbei Agile Coaches innerhalb der Unternehmen als methodische Unterstützung genannt. Diese sollen die detaillierten Kenntnisse zu möglichen Methoden, die bei der Einführung agiler Methoden unterstützen können, vermitteln und die beteiligten Mitarbeiter für diese sensibilisieren. Intervierpartner 4, ein Projektleiter aus der Prozessindustrie, unterstreicht die Bedeutung einer methodischen Unterstützung durch Agile Coaches auf folgende Art und Weise:

„Das wäre nicht nur hilfreich, das ist auch so. Es gibt ja die agilen Coaches, die genau dafür da sind. Die sind in den Meetings mit drinnen, stellen die Methodik vor, [...] wenn es dann mal ein bisschen auf die Beine gekommen ist, kommen die in mehr oder weniger regelmäßigen Abständen dazu, hören einfach mit zu und schlagen dann vielleicht was vor. [...]"

\section{Ergebnisse der quantitativen Untersuchung}

In der Qualitativen Studie wurde die Vermutung aufgeworfen, dass die Einführung von agilen Ansätzen angepasst an den Kontext erfolgen muss und einem individuellen Prozess folgt. Das Ziel der quantitativen Studie war es daher, die Notwendigkeit einer methodischen Einführung agiler Ansätze für eine erfolgreiche und nachhaltige Veränderung der Entwicklungsprozesse nachzuweisen. Dazu soll bestätigt werden, dass die Einführung agiler Ansätze aus Sicht von Mitarbeitern in Unternehmen und insbesondere in der Produktentwicklung einem Problemlösungsprozess folgt ( $\mathrm{H} 1)$. Zudem soll der Bedarf der Identifikation individueller Ziele zu Beginn des Prozesses, die mit der Einführung agiler Ansätze erreicht werden sollen, belegt werden (H2). Weiter soll gezeigt werden, dass für jeden Anwendungsfall ein spezifischer Ansatz abgeleitet werden soll (H3) und dieser gesamte Prozess methodisch gestützt werden sollte (H4).

Zu diesem Zweck wurde eine quantitative Umfrage erzeugt, die mittels 6 Fragen die Verifikation bzw. Falsifikation der Hypothesen ermöglicht. Diese wurde über zwei Karrierenetzwerke in entsprechenden Themengruppen verteilt. Zudem wurden persönliche Kontakte zur Beantwortung angefragt. Insgesamt haben 140 Teilnehmer den Fragebogen vollständig beantwortet. 


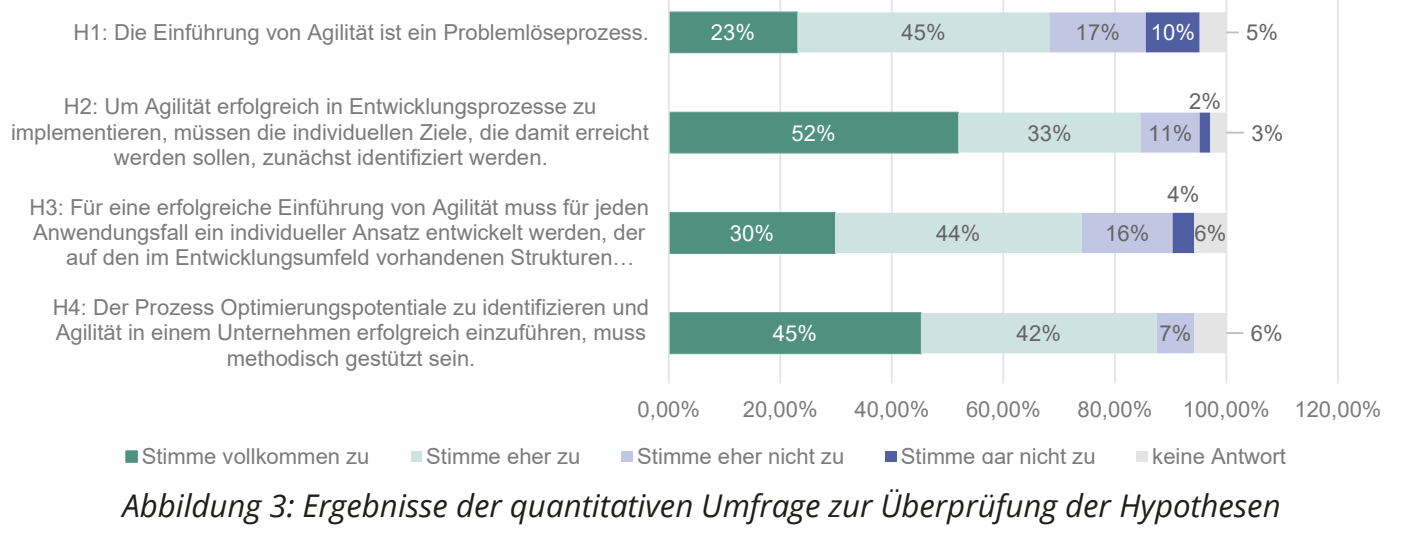

Der Hypothese H1, dass Agilität ein Problemlöseprozess ist, stimmten $71 \%(n=99)$ der gesamten Teilnehmer mindestens eher zu. Betrachten wir hier nur Teilnehmer, die sich als Experten im Bereich Agilität bezeichnen, vergrößert sich dieser Prozentsatz sogar auf $80 \%(n=25)$ in dieser Gruppe.

Deutlich höher lag die Zustimmung mit $87 \%(n=101)$ bei der Hypothese H2. Besonders interessant ist hierbei, dass nur $8 \%(n=50)$ der Produktentwickler hier nicht zustimmten.

Bezüglich der Hypothese H3 unterstützen $79 \%(n=98)$ der Teilnehmer die Autoren in ihrer Annahme. Betrachten wir hier nur diejenigen, die angaben, Experte im Bereich Agilität zu sein, steigt die Zustimmung sogar auf $84 \%(n=25)$.

Die zentrale Annahme H4 der Autoren, dass die Einführung von Agilität methodisch gestützt sein sollte, erfährt in dieser Umfrage mit $93 \%(n=98)$ die größte Zustimmung. Diese lag bei den Produktentwicklern sogar bei $94 \%(n=49)$ und bei agilitätsaffinen Teilnehmern ebenfalls bei $94 \%(n=67)$.

Schlussfolgernd ist zu sagen, dass in allen Hypothesen eine starke Tendenz zu erkennen ist, welche die Autoren in ihren Annahmen bestätigen. Alle Hypothesen übersteigen in dieser Umfrage den von den Autoren festgelegten kritischen Wert von $70 \%$. Auf Grund der großen Zustimmung werden somit alle Hypothesen von den Autoren als bestätigt anerkannt. 


\section{Zusammenfassung und Ausblick}

Agile Methoden und Vorgehensmodelle finden zunehmend Anwendung im Bereich der physischen Produktentwicklung. Dabei erfolgt die Einführung agiler Methoden in der Regel durch die anpassungsfreie Anwendung bestehender Ansätze, die vor allem in der Software-Entwicklung genutzt werden. Die Ergebnisse einer qualitativen und quantitativen Studie haben gezeigt, dass eine Einführung agiler Methoden individuell, unternehmensspezifisch sowie zielorientiert gestaltet werden muss, um die Vorteile agiler Methoden ausschöpfen zu können. Zudem konnte gezeigt werden, dass eine methodische Unterstützung bei der Einführung agiler Methoden hilfreich ist, um die Fülle an agilen Methoden und Vorgehensmodellen richtig einschätzen und schließlich auch effektiv und effizient in einem individuellen Unternehmenskontext anwenden zu können. Aufbauend auf diesen Erkenntnissen lassen sich individuelle Einführungskonzepte und eine methodische Unterstützung bei der Einführung agiler Methoden entwickeln.

\section{Literaturverzeichnis}

Albers, A., Heimicke, J., Spadinger, M., Reiss, N., Breitschuh, J., Richter, T. et al. (2019). Eine Systematik zur situationsadäquaten Mechatroniksystementwicklung durch ASD - Agile Systems Design. In KIT Scientific Working Papers (Bd. 113). Karlsruhe: KIT. https://doi.org/10.5445//R/1000091847

Albers, A., Heimicke, J., Walter, B., Basedow, G. N., Reiß, N., Heitger, N. et al. (2018). Product Profiles: Modelling customer benefits as a foundation to bring inventions to innovations. Procedia CIRP, 70(1), 253-258. https://doi.org/10.1016/j.procir.2018.02.044

Atzberger, A., Nicklas, S. J., Schrof, J., Weiss, S. \& Paetzold, K. (2020). Agile Entwicklung physischer Produkte. Universität der Bundeswehr München. https://doi.org/10.18726/2020_5

Cooper, R. G. (1990). Stage-gate systems: A new tool for managing new products. Business Horizons, 33(3), 44-54. https://doi.org/10.1016/0007-6813(90)90040-I

Cooper, R. G. (2014). What's Next? After Stage-Gate. Research-Technology Management, 57(1), 20-31. https://doi.org/10.5437/08956308X5606963

Cooper, R. G. \& Sommer, A. F. (2018). Agile-Stage-Gate for Manufacturers. Research-Technology Management, 61(2), 17-26. https://doi.org/10.1080/08956308.2018.1421380

Diebold, P. [Phillipp], Küpper, S. \& Zehler, T. (2015). Nachhaltige Agile Transition: Symbiose von technischer und kultureller Agilität. In M. Engstler, M. Fazal-Baqaie, E. Hanser, M. Mikusz \& A. Volland (Hrsg.), Projektmanagement und Vorgehensmodelle 2015 (S. 121-126). Bonn: Gesellschaft für Informatik e.V.

Goevert, K., Heimicke, J., Lindemann, U. \& Albers, A. (2019). Interview Study on the Agile Development of Mechatronic Systems. Proceedings of the Design Society: International Conference on Engineering Design, 1(1), 2287-2296.

https://doi.org/10.1017/dsi.2019.235 
Heimicke, J., Chen, R. \& Albers, A. (2020). AGILE MEETS PLAN-DRIVEN - HYBRID APPROACHES IN PRODUCT DEVELOPMENT: A SYSTEMATIC LITERATURE REVIEW. Proceedings of the Design Society: DESIGN Conference, 1, 577-586.

https://doi.org/10.1017/dsd.2020.259

Heimicke, J., Dühr, K., Krüger, M., Ng, G.- L. \& Albers, A. (2021). A Framework for Generating agile Methods for Product Development. In Proceedings of 31st CIRP Design Conference 2021 (in print).

Heimicke, J., Kaiser, S. \& Albers, A. (2021). Agile Product Development: An Analysis of Acceptance and Added Value in Practice. In Proceedings of 31 st CIRP Design Conference 2021 (in print).

Heimicke, J., Ng, G.- L., Krüger, M. \& Albers, A. (2021). A Systematic for Realizing Agile Principles in the Process of Mechatronic Systems Development through Individual Selection of Suitable Process Models, Development Methods and Practices. In Proceedings of 31st CIRP Design Conference 2021 (in print).

Karlström, D. \& Runeson, P. (2006). Integrating agile software development into stage-gate managed product development. Empirical Software Engineering, 11(2), 203-225. https://doi.org/10.1007/s10664-006-6402-8

Moreira, M. E. (2013). Being Agile. Your Roadmap to Successful Adoption of Agile. Berkeley, CA, s.l.: Apress. https://doi.org/10.1007/978-1-4302-5840-7

Naefe, P. \& Luderich, J. (2020). Konstruktionsmethodik für die Praxis. Aktuelle Verfahren in der Produktentwicklung (2., überarbeitete Auflage). Wiesbaden: Springer Vieweg.

Ovesen, N. (2012). The Challenges of becomming agile: IMPLMENTING AND CONDUCTING SCRUM IN INTEGRATED PRODUCT DEVELOPMENT. PhD Thesis. Aalborg University.

Petersen, K. \& Wohlin, C. (2010). The effect of moving from a plan-driven to an incremental software development approach with agile practices. Empirical Software Engineering, 15(6), 654-693. https://doi.org/10.1007/s10664-010-9136-6

Philipp Mayring \& Thomas Fenzl (2014). Qualitative Inhaltsanalyse. In Handbuch Methoden der empirischen Sozialforschung (S. 543-556). Springer VS, Wiesbaden. https://doi.org/10.1007/978-3-531-18939-0_38

Schwaber, K. \& Sutherland, J. (2017). The Scrum Guide. Zugriff am 09.11.2017.

Theobald, S. \& Diebold, P. [P.] (2018). Interface Problems of Agile in a Non-agile Environment. In J. Garbajosa, X. Wang \& A. Aguiar (Eds.), Agile Processes in Software Engineering and Extreme Programming. 19th International Conference, XP 2018, Porto, Portugal, May 21-25, 2018, Proceedings (Lecture Notes in Business Information Processing, vol. 314, vol. 314, S. 123-130). Cham: Springer International Publishing. https://doi.org/10.1007/978-3-319-91602-6_8

Richtlinie, VDI 2221 Blatt 1 (2019). Entwicklung technischer Produkte und Systeme. Berlin: Beuth.

Wessels, H., Heimicke, J., Rapp, S., Grauberger, P., Richter, T., Matthiesen, S. et al. (2019). Sprint planning in mechatronic system development based on reference system elements. https://doi.org/10.18154/RWTH-2019-08788 


\section{Kontakt}

Jonas Heimicke, M. Sc.

Ahmed Spahic, B. Sc.

Luis Bramato

Univ.-Prof. Dr.-Ing. Dr. h. c. Albert Albers

IPEK - Institut für Produktentwicklung am Karlsruher Institut für Technologie (KIT)

Kaiserstraße 10

76131 Karlsruhe

www.kit.edu 